\title{
Penile Chordee
}

National Cancer Institute

\section{Source}

National Cancer Institute. Penile Chordee. NCI Thesaurus. Code C122990.

Congenital curvature of the penis. 